\title{
BMJ Open Facilitating mental health help-seeking by young adults with a dedicated online program: a feasibility study of Link
}

\author{
Sylvia D Kauer, ${ }^{1}$ Kerrie Buhagiar, ${ }^{2}$ Victoria Blake, ${ }^{2}$ Sue Cotton, ${ }^{3,4}$ Lena Sanci ${ }^{1}$
}

To cite: Kauer SD, Buhagiar K, Blake $\mathrm{V}$, et al. Facilitating mental health help-seeking by young adults with a dedicated online program: a feasibility study of Link. BMJ Open 2017;7:e015303. doi:10.1136/ bmjopen-2016-015303

- Prepublication history and additional material are available. To view these files please visit the journal online (http://dx.doi. org/10.1136/bmjopen-2016015303).

Received 24 November 2016 Revised 25 February 2017 Accepted 6 April 2017

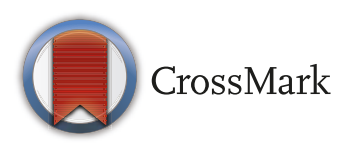

${ }^{1}$ Department of General Practice, The University of Melbourne, Carlton, Australia

${ }^{2}$ ReachOut Australia, Sydney, Australia

${ }^{3}$ Orygen, The National Centre of Excellence in Youth Mental Health, Parkville, Australia

${ }^{4}$ Centre for Youth Mental Health, The University of Melbourne, Parkville, Australia

Correspondence to

Dr Sylvia D Kauer; sylvia.kauer@ unimelb.edu.au

\section{ABSTRACT}

Objective To explore the feasibility of a dedicated online youth mental health help-seeking intervention and to evaluate using a randomised controlled trial (RCT) study design in order to identify any modifications needed before commencement of the full-scale RCT.

Design A pilot RCT with 1:1 randomisation to either the intervention or comparison arm.

Setting An online study conducted Australia-wide.

Participants $18-25$ year olds living in Australia were recruited via social media.

Intervention Link is a dedicated online mental health help-seeking navigation tool that matches user's mental health issues, severity and service-type preferences (online, phone and face-to-face) with appropriate youthfriendly services. The comparison arm was usual helpseeking strategies with a link to Google.com.

Main outcome measures The primary outcome was the number of acceptability and feasibility criteria successfully met. Intervention and study design acceptability and feasibility were assessed by nine criteria. Secondary outcomes, via online surveys (at baseline, 1 week and 1 month) measured service use, help-seeking intentions, psychological distress, barriers to help-seeking, attitudes towards mental health help-seeking, mental health literacy, satisfaction and trust.

Results Fifty-one participants were randomised (intervention: $n=24$; comparison: $n=27$ ). Three out of four of the intervention and two out of five of the study design criteria were met. Unmet criteria could be addressed by modifications to the study design. Qualitative analysis demonstrated that Link was useful to participants and may have increased their positive experiences towards helpseeking. There were no observable differences between arms in any outcome measures and no harms were detected.

Conclusion Generally, the Link intervention and study design were acceptable and feasible with modifications suggested for the four out of nine unmet criteria.

The main trial will hence have shorter surveys and a simpler recruitment process, use positive affect as the primary outcome and will not link to Google.com for the comparison arm.

Trial registration number Australian New Zealand Clinical Trials Registry, ACTRN12614000386639.

\section{INTRODUCTION}

Mental health disorders account for the highest burden of disease in adolescence
Strengths and limitations of this study

- This study provides important insights into the feasibility of a unique internet intervention for mental health help-seeking for young adults and the study design in preparation for a full-scale randomised controlled trial.

- The study uses social media as an innovative technique to recruit 18-24year olds across Australia, reflecting how young adults would learn about the intervention in a real-world scenario.

- Following the Medical Research Council's complex intervention guidelines, the intervention is underpinned by a sound theoretical framework ensuring that the design matches the intended goals.

- General practitioners, psychological experts, other service providers, technical experts and young adults were involved in a process of codesign to develop the Link intervention.

- This pilot study was not powered to assess the statistical significance of the outcomes and as such the results are descriptive, not generalisable, and should be interpreted with caution.

and young adulthood. ${ }^{1}$ Up to one quarter of young people experience mental health problems with substantial negative effects on interpersonal relationships, functioning at school and work, general health and wellbeing. ${ }^{2}$ While there has been significant investment in mental health service promotion and delivery over the last decade, ${ }^{3}$ only $35 \%$ of young people experiencing mental health problems seek professional help. ${ }^{45}$

The major barriers preventing young people from seeking help include lack of recognition of mental health problems, ${ }^{6}$ lack of awareness about appropriate mental health services, ${ }^{78}$ not being ready to seek help, ${ }^{2}$ lack of clinical detection ${ }^{9}$ and the stigma associated with mental illness and seeking professional help. ${ }^{10}$

Facilitated access to treatment services is necessary to improve mental health outcomes, ${ }^{11}$ particularly interventions aimed 
at increasing young people's willingness or readiness to seek help. ${ }^{12}$ The international Delphi panel on mental disorders suggested technological solutions may improve treatments, access to care and advance prevention and early intervention strategies. ${ }^{13}$ Young people find information and access mental health services on the internet and often prefer anonymous sources of help to traditional services. ${ }^{14-16}$ Yet, young people report that the help-seeking journey can be complex and repetitive. ${ }^{17}$ Non-dedicated search engines such as Google, while providing a vast array of information and sources, do not discern which services are appropriate for young people and may provide inaccurate and misleading advice and information. ${ }^{17}$ Furthermore, help-seeking outcomes associated with existing websites, search engines and dedicated online interventions are rarely evaluated. ${ }^{18} 19$

There is a paucity of evidence for online help seeking interventions and where there are studies, they are poorly designed. ${ }^{19}$ We sought to address this gap with a rigorous process, following the guidance of the Medical Research Council, involving an iterative approach across intervention development, feasibility testing and evaluation using a randomised trial, before further study on widespread implementation. ${ }^{20}$ Help-seeking is a complex behaviour to influence and designing an intervention aimed at improving help-seeking online must address multiple components of behaviour change including overcoming barriers to change, as well as providing algorithms to cluster user's symptoms into related condition categories and thereby direct users to appropriate services likely to meet their needs. Our intervention, Link, is an online program aimed at facilitating mental health help-seeking for young adults aged 18-25 years of age. Our prior development phase examined several theories of help-seeking and behaviour change to determine a suitable theoretical framework with the Theory of Planned Behaviour selected. ${ }^{21}$ Second, an iterative codesign process with young adults (end users), health service providers and clinical experts tailored the program for use with young adults seeking help for mental health issues. ${ }^{17}$

In this paper, we report on the feasibility study that aimed to: (1) assess the acceptability and feasibility of the online intervention, Link, to guide young people to appropriate mental healthcare or information; (2) assess the acceptability and feasibility of the study design for the future full-scale randomised controlled trial (RCT) evaluation; and (3) identify any modifications needed to the intervention or the study design before proceeding to the main trial. This pilot was essential to ensure that the intervention elements were acceptable, appropriate and functioned optimally and that the study design was feasible, including the recruitment and randomisation strategy, suitability of the outcome measures and choice of the primary outcome measure for assessing effectiveness and cost-effectiveness in the future RCT. This feasibility study was not powered to assess the statistical significance of changes in the intervention versus comparison arm and, as such, results are descriptive, not generalisable, and should be interpreted with caution.

\section{METHODS}

\section{Study design}

A two-arm RCT was undertaken. Individuals were baseline tested then randomised into balanced (1:1) parallel arms, the intervention arm (Link) or the comparison arm ('usual search strategies'), and then measured for primary and secondary outcomes with surveys at 1 week and 1 month postrandomisation. Online recruitment and online surveys were used. The online survey service, $\mathrm{QuON},{ }^{22}$ provided a secure server for managing the trial phases, issuing surveys and collecting data with responses deidentified and stored securely at the University of Newcastle, Australia. This study is reported following the CONSORT guidelines. ${ }^{23}$ The CONSORT checklist for the trial is provided in the online supplementary file.

\section{Participants}

\section{Recruitment}

Two recruitment methods were trialled using various social media and online platforms such as Google Ads, Facebook advertising and Gumtree: (1) a static advertising campaign with eight advertisements coupled with keywords and (2) a dynamic advertising campaign, where advertisements were regularly modified to maintain a high profile and visibility on the various platforms. Advertisements were limited to those living in Australia (for all platforms) and aged between 18 and 25 years (only Facebook allowed specification of age). This online recruitment strategy was used to reach the population most likely to use the intervention in the real world and to emulate how the intervention would be advertised if found to be effective. Online recruitment was chosen over traditional mass media or mail-out campaigns as it has been found to be as representative as mass-media campaigns. ${ }^{24}{ }^{25}$ Further advantages include being able to reach large numbers of participants quickly, a less confrontational format as there is no face-to-face contact and ease of participation with a click of a button. ${ }^{26}$ Recruitment using Facebook has been previously successful with this demographic. ${ }^{27}$

\section{Inclusion criteria}

In keeping with the intention of the Link intervention to provide a range of mental health service and information options according to a stepped care approach, ${ }^{28}$ the inclusion criteria were broad to encompass young adults with mild distress through to more severe mental health problems who have and have not previously sought help, and young people with or without clinical distress, seeking help for issues such as exam stress, relationship troubles, sleep or bullying. Young people had to be: (1) 18-25 years of age; (2) living in Australia; (3) able to provide three contact details such as a current valid email address and residential address and either a phone number or alternative email address; and (4) proficient enough in English to complete the surveys and intervention. Provision of 
a residential address ensured participants were living in Australia and allowed posting of gift vouchers for participation. The two other contact details were required to enable follow-up.

\section{Procedure}

Young people interested in the study clicked a link in the advertisements and were directed to the study website where a brief explanation of the study was provided along with check boxes confirming eligibility. Those eligible were directed to the information statement that fully explained the study objectives and procedures including the issuing of a $\$ A 25$ gift voucher on completion of the study. If interested in participating, eligible young people were asked to provide their contact details and to tick a box indicating consent to participation. The online system then issued an email to consenting participants instructing them to verify that the email address was correct. They were then directed to complete the baseline (prerandomisation) survey using their email address as a username to ensure participants could only participate once. Participants chose their own password. During this pilot, it became clear that many participants discontinued their participation at the email verification stage, and hence this step was removed (June 13th, 1 month into the pilot) with consenting participants instead progressing straight to the baseline survey. In addition, the information about gift vouchers was moved to the brief explanation page on which participants landed from the recruitment advertisements, in an effort to improve recruitment. Following the baseline survey, participants were randomised into either the intervention or comparison arm. One week and 1 month postrandomisation measurement surveys were conducted in both arms.

\section{Sample size}

Consistent with feasibility studies, no power analyses were conducted as this study was not intended to assess effectiveness of the intervention relative to the comparison arm. We anticipated that a total sample size of 120 participants (60 in each arm) would be sufficient to test acceptability and feasibility, allowing for approximately $50 \%$ attrition, as is common in online recruitment strategies, ${ }^{29}$ to ultimately reach a sample of 60 (30 in each arm) at the 1-week timepoint.

\section{Randomisation}

An independent academic oversaw the randomisation process including generating the sequence for the random allocation using a random seed generator within $\mathrm{QuON}$ in random blocks of four, six or eight. Participants were randomly allocated to a trial arm after baseline and identified by a randomly allocated identification number. The researchers were blind to the randomisation sequence until data analysis had been carried out. Participants were emailed a link to either Google.com (comparison) or Link (intervention), and therefore it was not feasible to blind participants.

\section{Interventions}

\section{Comparison arm: usual help-seeking strategies}

Participants allocated to the comparison arm received the following instructions: Please search for information and help for an issue you are currently facing using whatever strategies you would normally use whether it's online or offline. The below link will direct you to www.google.com to begin your search'.

\section{The Link intervention arm}

Participants allocated to the intervention arm were asked to seek help using Link, comprising three steps in a self-directed triage process (figure 1): (1) select issues (depression/anxiety, body image, drugs/alcohol, selfharm, suicide, bullying and relationship problems), (2) indicate severity on the Link severity scale (setting a level on an interactive pictorial sliding scale on a five-point scale to indicate level of severity from 'I'm OK', 'It's no big deal', 'It's a lot to handle', 'It's really tough' and 'It's a huge deal') and (3) select service preference (face-to-face, phone helpline, online chat or email therapy or online information).

Three services appropriate to these specified needs and preferences were then presented with the following information: a description of the service, what to expect, costs and a link for direct access to the service. A 'recommended' service, in addition to the participants' preferred service type, was also displayed based on clinical need. For example, a participant selecting suicidal thoughts as an issue with a preference for online help would also be recommended a 24-hour crisis telephone service ('Lifeline') in addition to their preferred service type. There were 31 youth-friendly services included in the Link directory. The program took a minimum of 30 s to complete (in order to reduce attrition during the program), but participants could spend longer exploring their issues or return at a later time to where they had ended a previous session if they wished. Intervention participants had access to Link for the duration of the trial (1 month in total). For the purposes of this study, participants accessed Link using their logon details to link their data with survey data. The development and prototype of the intervention is further described in another publication. ${ }^{21}$

\section{Measures}

\section{Primary outcome}

Based on previously used criteria, ${ }^{30}$ acceptability and feasibility of the intervention and study design are outlined in table 1; four criteria were used to assess the intervention and five criteria were used to assess the study design. Based on the number of criteria met, the study design or intervention were deemed 'not feasible' if no criteria were met, 'feasible with modifications' if some of the criteria were met, provided that modifications were possible, and 'feasible with no changes' if all the criteria were met. 


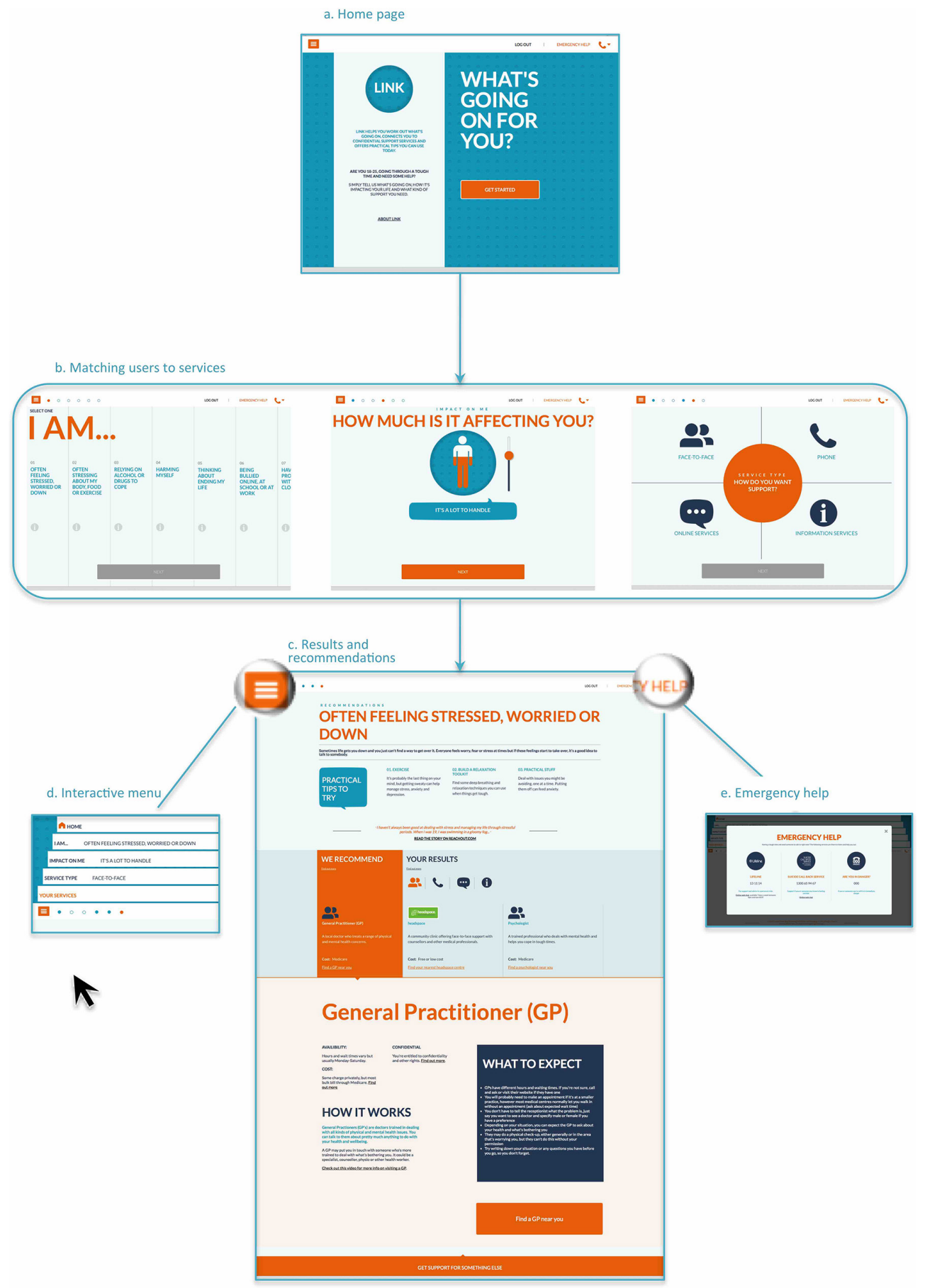

Figure 1 The webpages of Link.

\section{Secondary outcomes}

Help-seeking behaviours were assessed using the Mental Health Care Resource Use Questionnaire ${ }^{31}$; help-seeking intentions with the Stages of Change Questionnaire (range $=1-4$ ), with higher values indicating higher levels ${ }^{32}$; the General Help-Seeking Questionnaire (range 1-7), with higher values indicating increased likelihood of seeking help ${ }^{33}$; and two items created ('I want to seek help for my problems' and 'I intend to seek help for my problems)' using the Research-Based Education and Quality Improvement guidelines (range $=1-7$ ), with higher values indicating a higher level of intention. ${ }^{34} \mathrm{We}$ assessed mental health with the Kessler Psychological Distress Scale (K10) (range $=5-50)$, with higher values indicating higher psychological distress ${ }^{35}$; barriers to helpseeking with the Barriers to Adolescents Seeking Help Scale (range $=11-66$ ), with higher scores indicating more barriers ${ }^{736}$; quality of life with the Adolescent Quality of Life Scale ${ }^{37}$; mental health help-seeking perceptions with four created items (see online supplementary appendix A, supplementary figure A1) ranging from 4 to 28 , with higher scores indicating positive perceptions; and mental health literacy using the Mental Health Literacy Vignette. $^{38}$ 


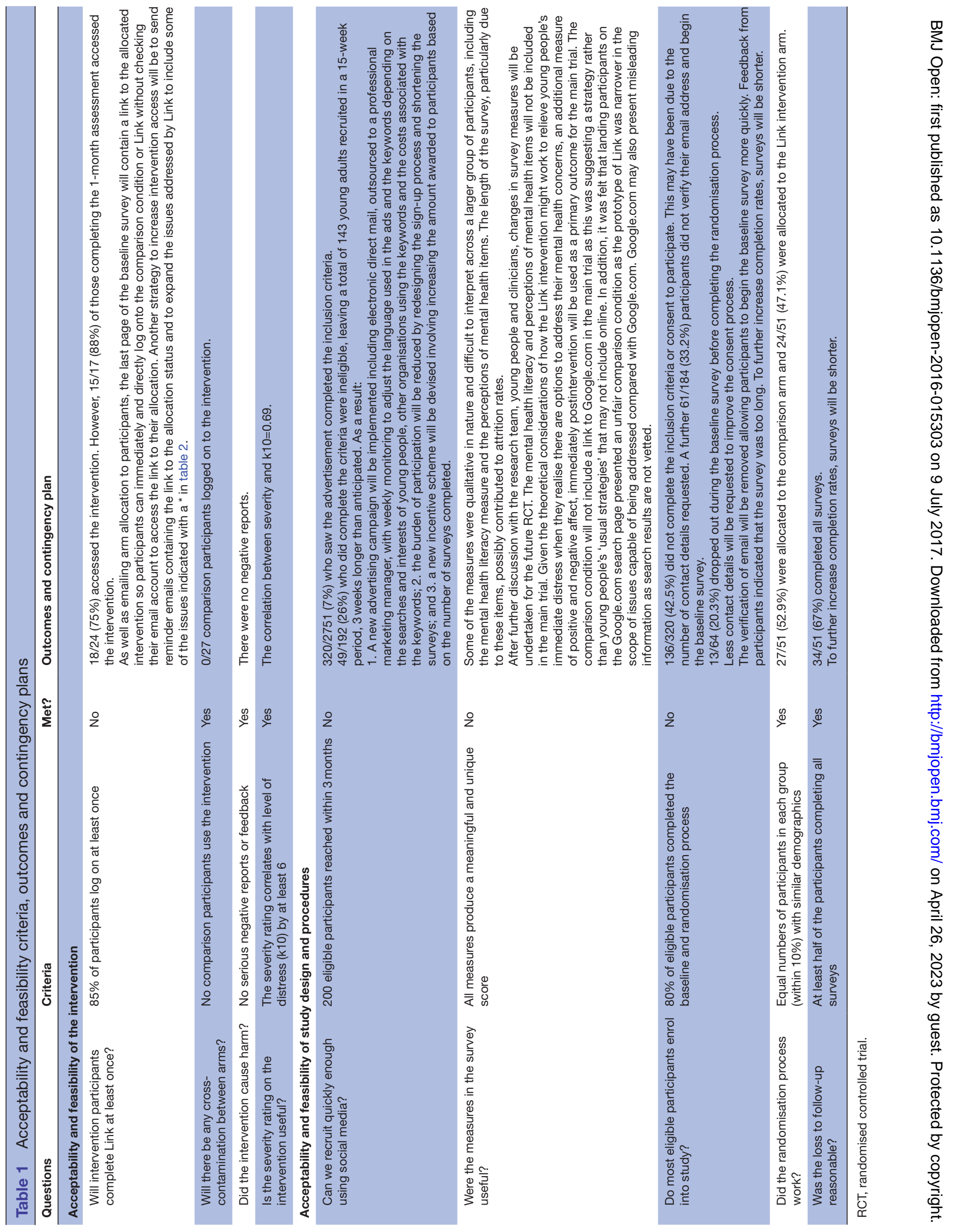


Further assessments at the 1 week and 1 month surveys included: satisfaction and trust in the service and the likelihood of using the service again using the Client Satisfaction Questionnaire (range $=8-40$ ), with higher scores indicating higher satisfaction ${ }^{39}$; whether expectations were met using 10 items adapted from Retolaza and Grandes (see online supplementary appendix A, supplementary figure A2) ranging from 10 to 50, with higher scores indicating more expectations met $^{40}$; and general feedback with four open-ended questions (What did you most like/dislike about the program? Do you have any suggestions for improvements? General comments).

The baseline surveys included all measures except for the questions about satisfaction and trust. The 1-week survey included only the questions about satisfaction and trust. The 1-month survey included all measures except measures of mental health literacy and perceptions.

\section{Data management and analysis}

Data from the online surveys and pathways through the Link intervention were collected into QuON. The Stata statistical package V.13 was used for data analysis. ${ }^{41}$ The primary outcome was the number of acceptability and feasibility items that were met. Descriptive statistics were used to describe the secondary outcomes. Effect sizes (Cohen's d) were calculated using bootstrapped SD for the satisfaction and trust questions. For all other continuous outcomes, multiple regression analyses were performed in order to calculate postestimation effect sizes; however, these are not reported due to the small sample size.

\section{RESULTS}

\section{Study procedure}

Figure 2 illustrates the participant flow throughout the study. The static advertising campaign ran from the 7 th May to 12th June 2014 with a total of 23 participants randomised during these 5 weeks. Next, the dynamic campaign was used from 13th June to 19th August 2014 with a total of 28 participants randomised within this 11-week period. The majority of participants $(45,88 \%)$ were recruited via Facebook advertising.

Of the 24 participants allocated to the intervention group, $18(75 \%)$ accessed Link. Only two participants (11.8\%) who completed the 1-month follow-up assessment did not access Link.

\section{Retention and assessment procedures}

Once participants were randomised into the study, attrition rates reduced. From the 51 randomised, only six participants (11.8\%; three from each arm) did not complete either of the surveys postintervention and were unable to be included in the outcome analysis. A further three $(5.9 \%)$ did not complete the 1 week survey and six (11.8\%) did not complete the 1 month survey, leaving a total of $34(66.7 \%)$ participants with complete data.

\section{Baseline data}

The mean age of participants was 20.9 years (intervention: $M=20.9, S D=2.1$; comparison: $M=21.0, S D=1.9)$. Other baseline characteristics are listed in table 2 by $\mathrm{arm}$. There were no differences between groups using $\chi^{2}$ test and independent samples t-test statistics.

Of the seven issues included in Link, participants reported that they were seeking help for: depression or anxiety, relationship problems, alcohol or drug use, bullying and body image. No participants mentioned self-harm or suicide. In addition, many participants also mentioned issues not covered in Link as listed in table 2. Thirteen participants $(21 \%)$ only described issues not covered in the program.

\section{Acceptability and feasibility of the intervention and study design}

The acceptability and feasibility outcomes are presented in table 1 with suggested modifications. Three of the four criteria were met for the intervention acceptability and feasibility criteria: no cross-contamination or adverse effects were reported, and the Link severity scale correlated with psychological distress (K10). The criterion of $85 \%$ of intervention participants logging onto Link was not met, with only $75 \%$ doing so. However, $88 \%$ of those completing the 1-month surveys logged onto Link, suggesting that failure to use Link may be a problem of engagement with the study measurement procedure. Therefore, the intervention was deemed feasible with minor modifications (listed in table 1) to the study design to increase the number of intervention participants using the intervention. It was also planned to expand the scope of pathways included in Link to address the other issues people described wanting assistance with (table 2).

For the study design, two out of five of the criteria were met: the randomisation process produced similar groups, and the expected completion rate was exceeded. The three criteria not met were the time it took to recruit participants, the usefulness of the measures used and enrolment into the study with minor modifications listed (table 1).

\section{Secondary outcomes}

Summary statistics of the measures used in the baseline and 1-month surveys are presented in table 3 in order to examine the acceptability and feasibility of using these measures in the main trial. No inferential statistics are reported due to the small sample size.

There were no notable differences between groups (partial $\boldsymbol{\eta}^{2}$ 20.1) except for a small effect in the precontemplation scale of the Stages of Change Questionnaire (partial $\boldsymbol{\eta}^{2} 20.4$ ), with favourable results for the comparison group. 
Viewed advertisement $(n=2751)$

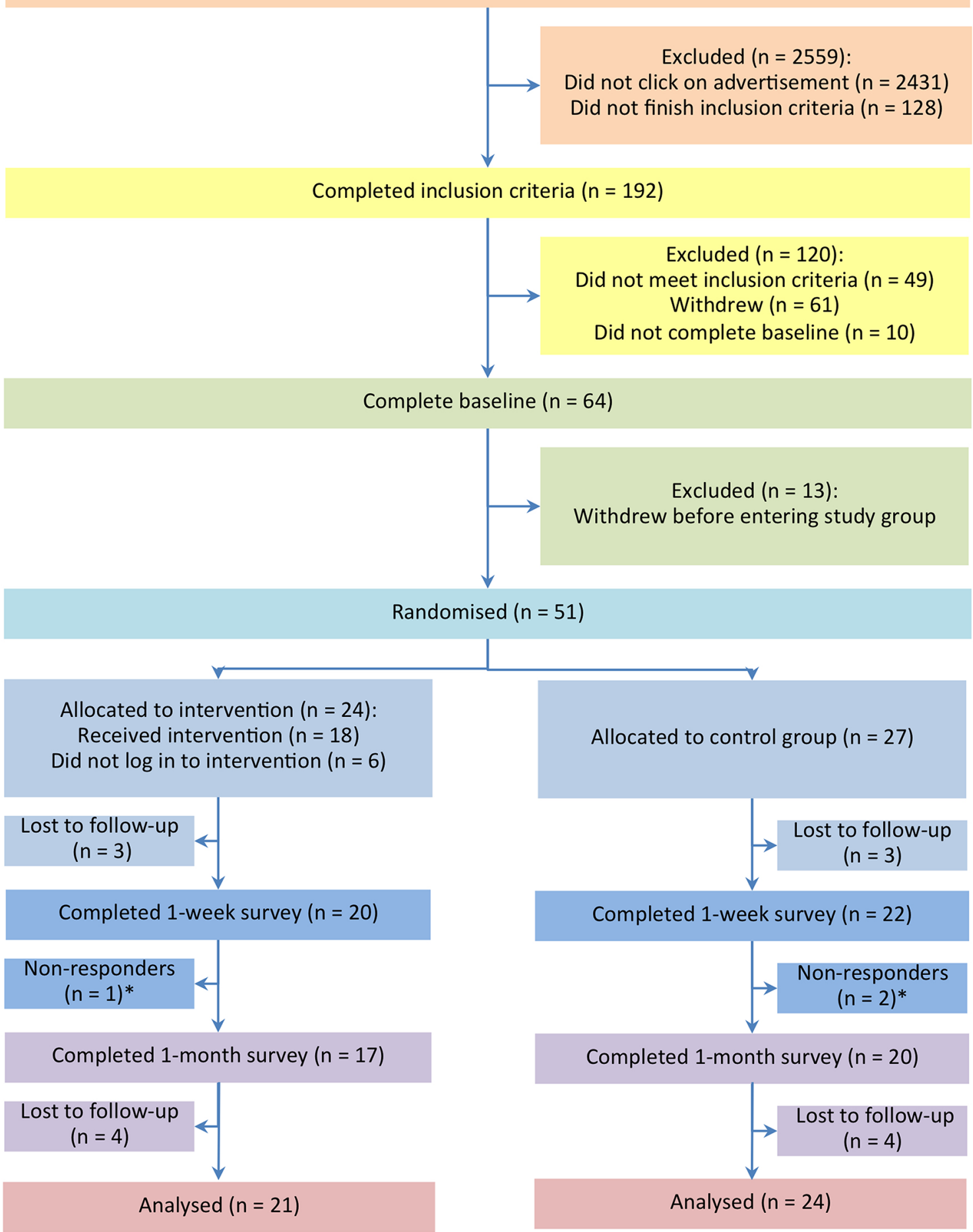

Figure 2 The study flow diagram. * These participants did not complete the 1-week survey but completed the 1-month survey. Note: Withdrew before entering study group=started baseline then withdrew, or completed baseline and withdrew before randomisation.

Satisfaction, trust and whether the participants' expectations were met for the help-seeking strategies are presented in table 4. Intervention participants reported more satisfaction, expectations met and trust at post-test compared with the comparison arm (small effect sizes), with a small effect size favouring the comparison group for satisfaction at 1 month. There were no differences between arms for expectations met or trust at 1-month follow-up.

The usefulness of the Link severity scale

We compared the Link severity scale with K10 scores. A positive linear relationship $(\mathrm{r}=0.81)$ between the Link severity scale and the baseline scores of K10 was found (figure 3). 
Table 2 Baseline characteristics by arm

\begin{tabular}{|c|c|c|c|}
\hline & Comparison $(n=27)$ & Link $(n=24)$ & Total $(n=51)$ \\
\hline & $n(\%)$ & $n(\%)$ & $n(\%)$ \\
\hline Female & $22(81.5)$ & $17(70.8)$ & 39 (76.5) \\
\hline Rural & $9(3.3)$ & $4(16.7)$ & $13(25.5)$ \\
\hline \multicolumn{4}{|l|}{ State } \\
\hline Australian Capital Territory & $4(14.8)$ & $1(4.2)$ & $5(9.8)$ \\
\hline New South Wales & $4(14.8)$ & $4(16.7)$ & $8(15.7)$ \\
\hline Queensland & $4(14.8)$ & $4(16.7)$ & $8(15.7)$ \\
\hline South Australia & 0 & $3(12.5)$ & $3(5.9)$ \\
\hline Victoria & $12(44.4)$ & $10(41.7)$ & $22(43.1)$ \\
\hline Western Australia & $3(11.1)$ & $2(8.3)$ & $5(9.8)$ \\
\hline Language other than English & $2(7.4)$ & $5(20.8)$ & $7(13.7)$ \\
\hline Completed higher education & $15(55.6)$ & $14(58.3)$ & $29(56.9)$ \\
\hline High distress (K10>19) & $21(77.8)$ & $18(75.0)$ & $39(76.5)$ \\
\hline Mental Health Literacy & $25(92.6)$ & $20(87.0)$ & $51(91.1)$ \\
\hline \multicolumn{4}{|l|}{ Issues } \\
\hline Depression & $14(51.9)$ & $19(79.2)$ & $33(64.7)$ \\
\hline Relationship problems & $12(44.4)$ & $9(37.5)$ & $21(41.2)$ \\
\hline Body image & $2(7.4)$ & $4(16.7)$ & $6(11.8)$ \\
\hline Alcohol/drug use & $2(7.4)$ & $1(4.2)$ & $3(5.9)$ \\
\hline Bullying & 0 & $2(8.3)$ & $2(3.9)$ \\
\hline University/school* & $10(37.0)$ & $12(50.0)$ & $22(43.1)$ \\
\hline Financial issues* & $5(18.5)$ & $10(41.7)$ & $15(29.4)$ \\
\hline Physical/chronic illness* & $2(7.4)$ & $6(25.0)$ & $8(15.7)$ \\
\hline Concerns about the future & $3(11.1)$ & $4(16.7)$ & $7(13.7)$ \\
\hline Employment issues ${ }^{*}$ & $5(18.5)$ & $4(16.7)$ & $9(17.6)$ \\
\hline Trauma* & $3(11.1)$ & $1(4.2)$ & $4(7.8)$ \\
\hline Other $^{\star}$ & $4(14.8)$ & $7(29.2)$ & $11(21.6)$ \\
\hline
\end{tabular}

*Participants' self-reported issues that were not listed in the intervention.

Note: More than one issue could be selected. Issues were coded from open-ended statements, hence participants could write as many issues as applied. The Other category included life and communication skills, concern for another person's well-being, obtaining a driver's licence, parenting and sexuality.

Further examination of the qualitative responses revealed that participants in the Link intervention arm thought it was a useful program. In particular, the majority of participants in the Link arm found that it was quick, easy, self-directed, personalised and had lots of resources.

Some participants had specific problems (eg, chronic illness, financial, pregnancy and worry about the future) that were not addressed in Link, commenting that the 'information was not appropriate' (female, aged 19), 'not understandable' (female, aged 21), 'impersonal' (females, aged 19 and 21) or 'too long' (female, aged 23). Lack of trust in the accuracy of the information available on the internet was also a general concern for both the Link and comparison arms.

\section{DISCUSSION}

This feasibility study piloted the acceptability and feasibility of the Link intervention and the study procedures to be used in the future RCT of Link, an online program to assist young adults seeking help for mental health problems. The results demonstrated that the study procedure, involving online surveys and the internet intervention, were feasible and acceptable with several minor modifications identified to enhance recruitment, intervention use and retention in the main trial. There were no indications that the Link or the Google arm caused harms in the participants.

\section{Acceptability and feasibility of the intervention and study design}

Of the nine acceptability and feasibility criteria, four (logging onto and completing the intervention, recruitment rates, usefulness of the surveys and enrolment rates) were not met. These outcomes were examined to determine likely modifications to successfully meet the criteria in the main trial and are described below. 
Table 3 Baseline and 1-month follow-up scores for help-seeking measures

\begin{tabular}{|c|c|c|c|c|}
\hline & \multicolumn{2}{|l|}{ Comparison } & \multicolumn{2}{|l|}{ Link } \\
\hline & Baseline $(n=27)$ & One month $(n=20)$ & Baseline $(n=24)$ & One month $(n=17)$ \\
\hline Resources & $n(\%)$ & $n(\%)$ & $n(\%)$ & $n(\%)$ \\
\hline Online & $11(40.70)$ & $14(70.00)$ & $8(33.30)$ & $8(47.10)$ \\
\hline None & $4(14.80)$ & $2(15.40)$ & $3(12.50)$ & $2(25.00)$ \\
\hline Distress (K10) & $27.6(9.36)$ & $25.4(9.78)$ & $28.1(9.07)$ & $24.4(7.06)$ \\
\hline \multicolumn{5}{|l|}{ General Help-Seeking Questionnaire } \\
\hline Total & $3.85(0.81)$ & $4.06(0.85)$ & $3.73(0.92)$ & $3.85(0.62)$ \\
\hline Professional & $3.69(1.29)$ & $3.88(1.42)$ & $3.26(1.53)$ & $3.47(1.21)$ \\
\hline Personal & $3.67(1.27)$ & $4.1(1.19)$ & $4.11(1.12)$ & $4.33(0.86)$ \\
\hline Precontemplation & $1.72(0.44)$ & $1.78(0.49)$ & $1.91(0.23)$ & $1.36(0.42)$ \\
\hline Contemplation & $2.73(0.39)$ & $2.65(0.38)$ & $2.64(0.45)$ & $2.65(0.41)$ \\
\hline Action & $3.03(0.52)$ & $2.9(0.66)$ & $3.23(0.75)$ & $3.51(0.40)$ \\
\hline Maintenance & $2.41(0.52)$ & $2.32(0.57)$ & $2.34(0.80)$ & $2.49(0.50)$ \\
\hline I want to seek help & $3.96(1.02)$ & $3.56(1.12)$ & $3.71(1.04)$ & $3.67(1.01)$ \\
\hline I intend to seek help & $4.15(1.14)$ & $3.7(1.17)$ & $3.71(1.40)$ & $3.41(1.12)$ \\
\hline Barriers to Adolescents Seeking Help & $37.6(7.39)$ & $36.93(7.32)$ & $34.7(10.10)$ & $34.94(6.91)$ \\
\hline Mental health help seeking perceptions & $17.5(2.44)$ & $17.9(1.46)$ & $17(2.19)$ & $17.94(2.36)$ \\
\hline
\end{tabular}

Will intervention participants complete Link at least once?

As the number of intervention participants who completed the intervention fell short of the expected number $(75 \%$ instead of $85 \%$ ), we will increase the visibility of the link to the intervention program in email reminders to participants to complete the program. Likewise, comparison participants will receive reminders to

Table 4 Participants' satisfaction, trust and whether their expectations were met

\begin{tabular}{llll}
\hline & $\begin{array}{l}\text { Comparison } \\
(\mathbf{n = 2 7})\end{array}$ & Link (n=24) & $\begin{array}{l}\text { Effect } \\
\text { size }\end{array}$ \\
\cline { 2 - 4 } & $\mathbf{M}(\mathbf{S D})$ & $\mathbf{M}(\mathbf{S D})$ & $\mathbf{d}^{*}$ \\
\hline One-week outcomes & & & \\
Satisfaction & $19.52(5.09)$ & $20.75(4.00)$ & 0.3 \\
Expectations met & $32.14(6.75)$ & $34.20(4.21)$ & 0.4 \\
Trust & $3.38(0.80)$ & $3.55(0.69)$ & 0.2 \\
One-month outcomes & & & \\
Satisfaction & $22.40(4.15)$ & $21.71(4.36)$ & 0.2 \\
Expectations met & $35.50(7.90)$ & $34.41(7.86)$ & 0.1 \\
Trust & $3.90(0.72)$ & $3.82(0.73)$ & 0.1 \\
\hline
\end{tabular}

${ }^{*}$ Effect sizes are based on bootstrapped SD. A value between 0.2 and 0.5 is considered a small effect. seek help using their usual strategies. As $88 \%$ of intervention participants who completed the 1-month survey also completed the intervention program, we anticipate that this minor modification will be sufficient to increase the number of intervention participants who access the Link intervention.

Can we recruit quickly enough using social media?

Two hundred 18-25year olds living in Australia were needed to ensure 60 who would participate in the study.

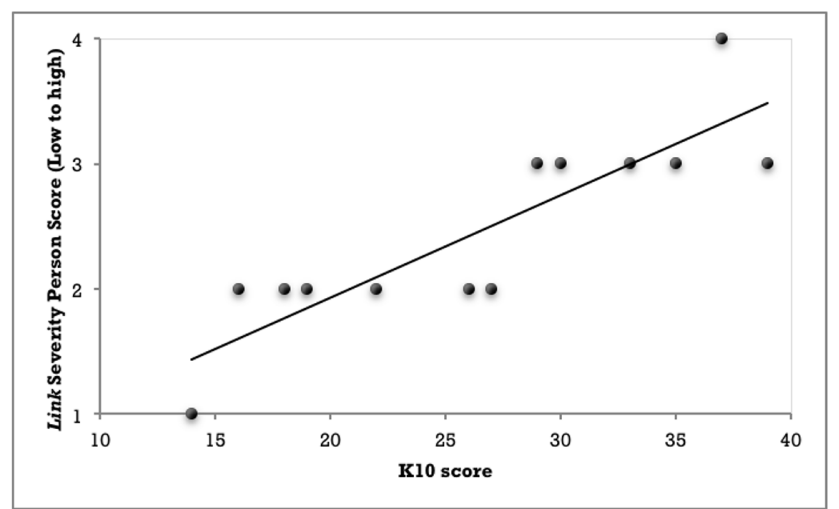

Figure 3 A scatterplot comparison of the Link severity scale with the baseline K10 scores with linear trend line. 
As the time frame for completing the study was limited by funding, it was necessary that this number was reached within 3 months. However, only 143 participants were recruited within a 15-week period. Therefore, two modifications will be implemented in the main trial to improve the recruitment rates: a new advertising campaign and a new reimbursement scheme.

\section{New advertising campaign}

Two methods of recruiting were trialled in this feasibility study, neither with much success. Further methods of online recruitment are needed to ensure an adequate sample size for the future main trial. Using email recruitment from young people signing up to targeted websites (such as ReachOut.com) may increase recruitment rates and will be explored for the main trial.

The dynamic nature of recruiting through Google and Facebook became apparent during the first weeks of our study. Due to the competitive nature of social media, flexible advertising with regular monitoring and adjustment is necessary to maintain visibility on social media streams. Increased flexibility of our advertising will increase exposure to young people and also reduce the costs associated with recruiting participants by choosing keywords that are not currently being used by other organisations. As advertising on social media is dynamic, and dependent on other organisations' use of keywords, young people's interests and searches as well as the costs associated with advertising, it is important we respond to the competitive nature of this advertising and have a dynamic and flexible advertising strategy with the ability to change the wording regularly and quickly. As this is a complex and time-consuming process, we will outsource this recruitment to a professional marketing manager with expertise in marketing and recruitment for research studies.

\section{New reimbursement scheme}

Alexander $e t$ al suggest that a small upfront payment with a higher incentive awarded for retention can increase recruitment and retention rates. ${ }^{42} \mathrm{~A} \$ \mathrm{~A} 25$ gift card was given to each participant who completed the 1-month survey. In order to reduce attrition rates early in the study procedure, rather than the $\$$ A25 gift card received at the end of the study, an incremental reimbursement schedule will be implemented with participants receiving increasing amounts per survey (eg, $\$ 10$ for baseline, $\$ 15$ after postintervention survey and $\$ 25$ after a 1-month follow-up survey).

\section{Were the measures in the survey useful?}

We included a large number of measures in the feasibility trial to explore their usefulness with the understanding that only the most relevant would be retained in the main trial. We found that the K10, the General Help-Seeking Questionnaire, the Stages of Change Questionnaire, the Barriers to Adolescents Seeking Help Scale, the Adolescent Quality of Life Scale and the Client Satisfaction Questionnaire were the most meaningful. Qualitative analyses suggest that participants found the surveys too time-consuming, therefore shortening the surveys is likely to increase completion rates. Therefore, several measures that provided less meaningful results will be removed for the larger trial including the mental health literacy scale and the items we created based on the Research-Based Education and Quality Improvement guidelines. ${ }^{34}$ These measures are not well validated and are not primary outcomes so removing them is anticipated to improve the study design and retention.

One of the benefits reported by participants in the qualitative responses was the immediate increase in positive affect after seeking help using Link. This outcome was also discussed as a likely first step in engaging young people with a help-seeking journey during participatory workshops with service providers, researchers and young people. Therefore, the Positive and Negative Affect Schedule will be used to assess positive affect after participants have been exposed to their intervention, as the primary outcome. ${ }^{43}$

\section{Do most eligible participants enrol into study?}

There was high attrition early in the study procedure indicating that methods to increase retention rates during enrolment into the study are necessary. First, the sign up process was time-consuming and participants may have had concerns about their privacy. Hence, an email address and a phone number only will be required for the main trial. Second, participants were asked to validate the survey on their email account before obtaining access to the baseline survey in the beginning of the pilot study. One of the changes made during the pilot study was a direct link to the baseline survey without the email validation process. This may reduce drop-off at this point. Third, $20 \%$ of participants $(n=13)$ dropped out after completing the baseline survey and before completing the randomisation process, indicating that processes to encourage participants to continue with the study are important here as well. The language used, length of the survey and the look of the website may have influenced the retention rates at this step. Further methods are needed to increase these rates such as including regular reminders via email and SMS.

\section{The Link severity scale}

Of interest, this study presents some validation for the severity scale used in Link, as it consistently correlated with scores on the K10. This suggests that the Link severity scale may be a valid measure of the impact of mental health problems on daily life and therefore will be retained within the Link intervention.

\section{Secondary outcomes}

As discussed above, several measures will be removed from the larger trial and the outcome of positive affect will be used as a primary outcome. Some of the current measures used (Stages of Change Questionnaire, Barriers to Adolescents Seeking Help Scale and General Help 
Seeking Questionnaire) are not well validated and therefore, while useful as secondary outcomes, are not suitable as a primary outcome. The Mental Health Care Resource Use Questionnaire was also useful and necessary as a secondary outcome; however, as the aim of Link is to direct young people to services appropriate to their needs and preferences, it is difficult to interpret from the services used whether this has occurred. An increase in service use is not necessarily useful for participants with low mental healthcare needs for example.

One of the benefits of online help-seeking strategies is that information is immediately available and the sense of relief felt by participants once an avenue of help is suggested. Participants indicated that they found Link helpful and easy to use. Providing avenues of care to participants may increase positive emotions and broaden personal resources for coping and help-seeking. Some of the features of Link based on the Theory of Planned Behaviour also tap into the concept of positive psychology. ${ }^{44}$ Increasing mental health literacy and providing young people with a sense of empowerment and meaning improves coping skills, ${ }^{44}$ particularly problem-focused coping such as help-seeking. By providing avenues for help-seeking in Link, in line with the Theory of Planned Behaviour, positive emotions are likely to occur leading to a positive experience of seeking help. Therefore, we expect that positive affect will be an appropriate primary outcome. ${ }^{43}$ This measure is well-validated and likely to mediate the relationship between beliefs, and help-seeking intentions and behaviours.

Many coping strategies that increase positive affect are reinforced in Link such as: positive reappraisal, goal-directed problem-focused coping, increasing the repertoire of coping strategies, relaxation and behavioural therapies and increasing positive meaning of issues. ${ }^{44-46}$ In line with this theory, Link aims to increase connections with others, improve distraction skills and increase self-esteem. Furthermore, positive emotions also improve coping skills and increase the likelihood of future emotional well-being. ${ }^{47}$

\section{Strengths and limitations}

Conducting this feasibility study was a useful way to optimise the intervention and the study design with many issues uncovered before commencement of the main RCT. There were many strengths including the novel recruitment strategies employed allowing a broad range of young adults to be involved in the study. The online survey, data management and randomisation were generally successful with few issues found.

While no differences between arms were anticipated due to the exploratory nature of this study, providing comparison participants with the link to Google was a limitation as it may have suggested this avenue of helpseeking and led participants to a help-seeking method not ordinarily in their repertoire. Furthermore, as Google is a well-developed program with years of programming to perfect the search engine, it is highly advanced compared with the Link prototype, which currently only maps pathways for seven issues and includes 31 services. Therefore, the comparison arm in the larger trial will exclude the link to Google and instead direct comparison participants to use their usual strategies for seeking help.

A measure of positive and negative affect was not included in this study. This outcome is a key element to mental health help-seeking, as positive affect associated with the act of help-seeking is important to continued help-seeking behaviour and obtaining help if needed. Many of the outcome measures used are not well validated or widely used. This is in part due to the small number of publications in the field, but also because of the complexity of mental health 'help-seeking' as a focus compared with mental health or psychological distress.

\section{Conclusions}

In this feasibility study, we found that the proposed study design and the Link intervention were feasible and acceptable to participants with some modifications. These will mainly include improving the recruitment strategies, lessening the burden on participants during sign-up and by shortening the surveys, choosing a different primary outcome measure to determine positive affect and using a more realistic comparison condition to elicit 'usual helpseeking strategies'. These are important features and processes to consider in developing and implementing a complex intervention. It is difficult to determine from this pilot trial whether Link will effectively improve helpseeking or positive affect; however, incorporating the improvements identified as a result of this study, the main randomised controlled trial will allow us to investigate the effects of Link on positive affect and help-seeking.

Acknowledgements This was a joint contribution of the Department of General Practice and ReachOut.com. Our research team included project managers Julie Grey and Andrea Browne and support staff Ann-Maree Duncan.

Contributors SDK prepared the materials, analysed the data and drafted the article. LS and KB obtained the funding. KB and VB oversaw the recruitment process. SC supervised the study design and statistical analyses. All authors were involved with the study design, critically reviewed the drafts and approved the submitted manuscript.

Funding Funding was from the Young and Well Cooperative Research Centre, an Australian-based international research centre that unites young people with researchers, practitioners, innovators and policymakers from over 70 partner organisations. SC is supported by a National Health and Medical Research Council (NHMRC) Career Development Fellowship (CDF).

Competing interests None declared.

Ethics approval This study was approved by the University of Melbourne Human Research Ethics Committee, reference no. 1341063.4. All participants consented to take part in this study via an online consent form.

Provenance and peer review Not commissioned; externally peer reviewed.

Data sharing statement No additional data are available.

Open Access This is an Open Access article distributed in accordance with the Creative Commons Attribution Non Commercial (CC BY-NC 4.0) license, which permits others to distribute, remix, adapt, build upon this work non-commercially, and license their derivative works on different terms, provided the original work is properly cited and the use is non-commercial. See: http://creativecommons.org/ licenses/by-nc/4.0/ 
(c) Article author(s) (or their employer(s) unless otherwise stated in the text of the article) 2017. All rights reserved. No commercial use is permitted unless otherwise expressly granted.

\section{REFERENCES}

1. Whiteford HA, Degenhardt L, Rehm J, et al. Global burden of disease attributable to mental and substance use disorders: findings from the global burden of disease study 2010. Lancet 2013;382:1575-86.

2. Tanielian T, Jaycox LH, Paddock SM, et al. Improving treatment seeking among adolescents with depression: understanding readiness for treatment. J Adolesc Health 2009;45:490-8.

3. Hickie IB, McGorry PD. Increased access to evidence-based primary mental health care: will the implementation match the rhetoric? Med $J$ Aust 2007;187:100-3.

4. King RK, Bickman L, Shochet I, et al. Use of the internet for provision of better counselling and psychotherapy services to young people, their families and carers. Psychother Aust 2010;17:66-74.

5. Andrews $\mathrm{G}$, Issakidis $\mathrm{C}$, Carter $\mathrm{G}$. Shortfall in mental health service utilisation. Br J Psychiatry 2001;179:417-25.

6. Jorm AF. Mental health literacy. Public knowledge and beliefs about mental disorders. Br J Psychiatry 2000;177:396-401.

7. Kuhl J, Jarkon-Horlick L, Morrissey RF. Measuring barriers to HelpSeeking behavior in adolescents. J Youth Adolesc 1997;26:637-50.

8. Tylee A, Haller DM, Graham T, et al. Youth-friendly primary-care services: how are we doing and what more needs to be done? Lancet 2007;369:1565-73.

9. Hickie IB, Fogarty AS, Davenport TA, et al. Responding to experiences of young people with common mental health problems attending Australian general practice. Med J Aust 2007;187:S47-52.

10. Clement S, Schauman O, Graham T, et al. What is the impact of mental health-related stigma on help-seeking? A systematic review of quantitative and qualitative studies. Psychol Med 2015:1-45.

11. Sanci L, Lewis D, Patton G. Detecting emotional disorder in young people in primary care. Curr Opin Psychiatry 2010;23:318-23.

12. Haller DM, Sanci LA, Sawyer SM, et al. The identification of young people' s emotional distress : a study in primary care. Br J Gen Pract 2009;70:61.

13. Collins PY, Patel V, Joestl SS, et al. Grand challenges in global mental health. Nature 2011;475:27-30.

14. Burns JM, Davenport TA, Durkin LA, et al. The internet as a setting for mental health service utilisation by young people. Med J Aust 2010;192:S22-6.

15. Blanchard M, Hosie A, Burns J. Embracing technologies to improve well-being for young people an Australian view of evidence and policy implications. Nexus Strateg Partnerships Commonw Secr 2013:127-32.

16. Rickwood DJ, McKenzie DP, Wilson CJ, et al. Young people's helpseeking for mental health problems. Aust e-Journal Adv Ment Heal 2005;4:1-35

17. Blake V, Buhagiar K, Kauer SD, et al. Using participatory design to engage young people in the development of a new online tool to increase help-seeking. J App/ Youth Stud 2016;1:68-83.

18. Cline RJ, Haynes KM. Consumer health information seeking on the Internet: the state of the art. Health Educ Res 2001;16:671-92.

19. Kauer SD, Mangan C, Sanci L. Do online mental health services improve help-seeking for young people? A systematic review. J Med Internet Res 2014;16:e66.

20. Craig P, Dieppe P, Macintyre S, et al. Medical Research Council Guidance. Developing and evaluating complex interventions: the new Medical Research Council guidance. BMJ 2008;337:a1655.

21. Kauer SD, Buhagiar K. Facilitating mental health help seeking in young adults: the underlying theory and development of an online navigation tool. Adv Ment Heal 2016.

22. Paul D, Nolan K, Koller C. QuON ANDS Research Group University of Newcastle Survey System. 2007 https://quon.newcastle.edu.au/ quon_doc/.

23. Altman DG, Schulz KF, Moher D, et al. CONSORT GROUP (Consolidated Standards of Reporting Trials). The revised CONSORT statement for reporting randomized trials: explanation and elaboration. Ann Intern Med 2001;134:663-94.

24. Treweek S, Lockhart P, Pitkethly M, et al. Methods to improve recruitment to randomised controlled trials: cochrane systematic review and meta-analysis. BMJ Open 2013;3:e002360.

25. Jones RB, Goldsmith L, Hewson P, et al. Recruitment to online therapies for depression: pilot cluster randomized controlled trial. $J$ Med Internet Res 2013;15:e45.

26. Murray E, Khadjesari Z, White IR, et al. Methodological challenges in online trials. J Med Internet Res 2009;11:e9.

27. Ramo DE, Prochaska JJ. Broad reach and targeted recruitment using Facebook for an online survey of young adult substance use. J Med Internet Res 2012;14:e28.

28. van Straten A, Seekles W, van 't Veer-Tazelaar NJ, et al. Stepped care for depression in primary care: what should be offered and how? Med J Aust 2010;192:S36-9.

29. Christensen H, Griffiths KM, Farrer L. Adherence in internet interventions for anxiety and depression. J Med Internet Res 2009;11:e13-16.

30. Als LC, Nadel S, Cooper M, et al. A supported psychoeducational intervention to improve family mental health following discharge from paediatric intensive care: feasibility and pilot randomised controlled trial. BMJ Open 2015:5:e009581.

31. Mihalopoulos C, Lee K, Magnus A, et al. The mental health care resource use questionnaire. Burwood, Melbourne, Australia 2014.

32. Lewis $C C$, Simons AD, Silva SG, et al. The role of readiness to change in response to treatment of adolescent depression. J Consult Clin Psychol 2009;77:422-8.

33. Wilson CJ, Deane FP, Ciarrochi J, et al. Measuring help-seeking intentions: properties of the General Help-Seeking Questionnaire. Can J Couns 2005;39:15-28.

34. Francis JJ, Eccles MP, Johnston M, et al. Constructing questionnaires based on the theory of Planned Behaviour: a manual for health services researchers. Newcastle, UK 2004.

35. Kessler RC, Andrews G, Colpe LJ, et al. Short screening scales to monitor population prevalences and trends in non-specific psychological distress. Psychol Med 2002;32:959-76.

36. Wilson CJ, Rickwood DJ, Ciarrochi J, et al. Adolescent barriers to seeking professional psychological help for personal-emotional and suicidal problems. In: 9th annual conference for suicide prevention Australia 2002.

37. Hawthorne G, Korn S, Richardson J. Population norms for the AQoL derived from the 2007 Australian National Survey of Mental Health and Wellbeing. Aust N Z J Public Health 2013;37:7-16.

38. Jorm AF, Wright A, Morgan AJ. Beliefs about appropriate first aid for young people with mental disorders: findings from an Australian national survey of youth and parents. Early Interv Psychiatry 2007;1:61-70.

39. Larsen DL, Attkisson CC, Hargreaves WA, et al. Assessment of client/patient satisfaction: development of a general scale. Eval Program Plann 1979;2:197-207.

40. Retolaza A, Grandes G. [Expectations and satisfaction in mental health center users]. Actas Esp Psiquiatr 2003;31:171-6.

41. StataCorp. Stata user's guide: release 13. College Station, TX: StataCorp LP, 2013.

42. Alexander GL, Divine GW, Couper MP, et al. Effect of incentives and mailing features on online health program enrollment. Am J Prev Med 2008;34:382-8

43. Crawford JR, Henry JD. The positive and negative affect schedule (PANAS): construct validity, measurement properties and normative data in a large non-clinical sample. $\mathrm{Br} \mathrm{J}$ Clin Psychol 2004;43:245-65.

44. Fredrickson BL. Cultivating positive emotions to optimize health and well-being. Prev Treat 2000;3:1-25

45. Folkman S, Moskowitz JT. Positive affect and the other side of coping. Am Psychol 2000;55:647-54

46. Fredrickson BL. The role of positive emotions in positive psychology. Am Psychol 2001;56:218-26.

47. Fredrickson BL, Joiner T. Positive emotions trigger upward spirals toward emotional well-being. Psychol Sci 2002;13:172-5. 